\title{
THE LARGE SCALE MICROWAVE BACKGROUND ANISOTROPY
}

IN DECAYING FARTICLE COSMOLOGY

\author{
Mirosław Panek 1 \\ NASA/Fermilab Astrophysics Group \\ P.O.Box 500, Batavia, II 60510
}

Abstract :

We investigate the large-scale anisotropy of the microwave background radiation in cosmological models with atcaying particles. The observed value of the quadrupole moment combined with other constraints gives an upper limit on the redshift of the decay $z_{d}<3-5$.

1 permanent adaress: Copernicus Astronomical Center, Bartycka 18 , D0-716 Warsaw, Poland 


\section{INTRODUCTION}

The Inflationary Universe models predict the $a=1$ universe. Unfortunately this useful and esthetic feature seems to be in contradiction with observations of the large-scale structure of the universe suggesting that $\Omega \approx 0.1-0.3$. One of the ways to resolve this problem is to assume that the universe is dominated today by the relativistic matter and the measured value of $\Omega$ is relevant only to nonrelativistic components. However, to ensure sufficient growth of density perturbations the universe had to be matter-dominated for some time. This leads to suggestion that the uriverse became radiation-dominated only recently.

The Decaying Particle Cosmology (DPC) (Turner, Steigman and Krauss 1984, Turner 1985a) assumes that in the past the universe was dominated by the nonrelativistic particles $x$ that at the redshift $z_{d}$ decayed into light, relativistic particles $R$ that are today smoothly distributed on scales corresponiing to the observations determining the value of $\Omega$.

An important constraint on this model comes from the observed age of globular clusters - the value of the Hubble constant today $H_{0}=h .100 \mathrm{~km} / \mathrm{s} \cdot \mathrm{Mpc}$ has to be $\mathrm{h} \leqslant 0.5$. This is a severe constraint, however even lower values of $\mathrm{h}$ are still suggested by some observations.

Vittorio and silk (1985) investigated the fine-scale microwave background radiation (MBR) anisotropies in DPC models with decaying neutrinos and found that they may be consistent with observations if $2 \leqslant z_{d} \leqslant 4, \quad 0.4 \leqslant h \leqslant 0.5$, $10 \cdot 10^{9} \mathrm{yr} \leqslant t_{0} \leqslant 12 \cdot 10^{9} \mathrm{yr}$ where $t_{0}$ is the age of the universe. Analogously, Turner $(1985 \mathrm{~b})$ found that if decaying particles were cold relics the value of the redshift of decay might be $z \leqslant 10$. Adopting the other normalization of the spectrum of perturbations Kolb, Olive and Vittorio (1986) obtained the limit $2 \leqslant z \leqslant 4$ being valid also for cold relics. 
In this paper we investigate the large-scale MBR anisotropy in DPC models of decaying neutrinos and decaying cold matter. We find the values of quadrupoie moment using the gauge-invariant formalism (Panek 1986). Comparing these values with the observed $a_{2}<10^{-4}$ we conlude that the redshift of decay must be $z_{d} \leqslant 3-5$ where the uncertainity comes from the method of normalization of the perturbation spectrum. One of these methods leads even to the conclusion that the neutrinos should be excluded as decaying particle candidates.

In Sec.II we descibe the equations of the evolution of perturbations and the formalism to obtain the MBR anisotropy. Sec.III describes the methods of normalization of the perturbation spectrum. Sec.IV contains the results and, finaliy, Sec.v. - conclusions.

II. THE EVOLUTION OF MODEL AND THE MBR ANISOTROPY

A. The evolution of the background.

we assume that after early, radiation-dominated stage the universe is filled with three forms of matter: stable, nonrelativistic particies NR (baryons + others); aominating for most of the matter-dominated era nonreiativistic, unstable particles $x$ that decay into light, relativistic particles $R$ that make the universe radiation-dominated again. Parameters that describe the model are $h, \delta_{N R}$ and $z_{d}$.

According to previously obtained constraints and observations the allowed range of $h$ in the model is $0.3-0.5$.

$\Omega_{j}=8 \pi \mathrm{G}_{j} / 3 \mathrm{H}_{0}^{2}$ is the ratio of density in form of the $j$ particles and the closure density taken today and $u_{N R}=0.0-0.3$ (for the purpose of calculations we take the value 0.1 ). We 
$-4-$

have $\delta_{X}+\delta_{R}+\Omega_{N R}=1$.

The considered range of values of $z_{\mathrm{d}}$ is $2-10$. The $x$ particles decay according to the exponential law and $z_{d}$ is defined as:

$$
z_{d}+1=\frac{\Omega_{N R}}{\Omega_{R}} \beta^{-1}, \quad \beta=\left(\frac{Q_{N R}}{Q_{x}}\right)_{i}
$$

where the subscript $i$ means that the ratio of densities is taken at some time $t_{i}$ at the beginning of the matter-dominated era.

The Einstein equations give us the evolution of the mosel. We define (Turner 1985a):

$$
\begin{array}{ll}
x=\Gamma t & f_{x}=\frac{Q_{x}}{Q_{x_{i}}} \\
H_{i}^{2}=\frac{8}{3} \pi G Q_{x_{i}} & f_{R}=\frac{Q_{R}}{Q_{x_{i}}} \\
x_{H}=\frac{\Gamma}{H_{i}} & f_{N R}=\frac{Q_{N R}}{Q_{x_{i}}}
\end{array}
$$

where $I=x_{H^{H}}{ }^{H}\left[\Omega_{N R} S_{R^{B}}^{3}{ }^{-1}(1+B)\right]^{1 / 2}$ is the $x^{-s}$ decay width and $S_{R}$ is the expansion parameter today $\left(S_{i}=1\right)$. "xi is the density of $X$ particles at the initial moment and is expressed in the choose model parameters as $\mathrm{XI}_{1}=3 \mathrm{H}_{0}^{2} \mathrm{rNR}_{\mathrm{N}} \mathrm{s}^{3}$. The evolution of the background is given by:

$$
\begin{gathered}
f_{x}=S^{-3} \exp (-x) \\
f_{R}=S^{-4} \int_{0}^{x} S\left(x^{\prime}\right) \exp \left(-x^{\prime}\right) d x^{\prime}
\end{gathered}
$$


$-5-$

$$
\begin{gathered}
f_{N R}=\beta S^{-3} \\
\frac{S^{\prime}}{S}=\left(f_{x}+f_{R}+f_{N R}\right)^{1 / 2}(1+\beta)^{-1 / 2} x_{H}^{-1}
\end{gathered}
$$

where prime denotes the derivative with respect to the new time variable $x$.

We have integrated these equations from some sufficiently small value of $x_{i}$ until when $f_{R^{\prime}} / f_{N R}=\left(1-\sigma_{N R}\right) / \sigma_{N R}$ which is identified with the present moment.

B. The evolution of perturbations.

To obtain the equations for the perturbations of matter we assumed that the NR particles follow the perturbations of $x$ particles during the matter-dominated era: $\varepsilon_{N R}=\varepsilon_{b}=\varepsilon_{x}$ (from now on we will use $\varepsilon_{b}$ because the MBF fluctuations are expressed in baryonic quantities). The perturbations of the $R$ particles on scales smaller than the horizon will be damped because of the free-streaming (these particles are most likely to be collisionless). However, the $R$ perturbations on scales larger than horizon are nonzero and we cannot neglect them, especially because we know that the most power to quadrupole moment comes from the scales comparable to the size of the horizon today.

The equations of evolution for perturbations of NR matter are:

$$
\left.\dot{\varepsilon}_{0}^{1}+\frac{3 q_{x_{i}}}{2 k^{2}} \mid f_{N R}+f_{x}+\frac{4}{3} f_{R}\right)_{u}+\frac{4}{S^{2}}+\frac{3}{s}\left|S \Phi_{H}\right|^{\prime}=0
$$




$$
\begin{aligned}
& u^{\prime}=-\frac{k^{2}}{r^{2}} \Phi_{H} \\
& \Phi_{H}=\frac{q_{x_{i}} S^{2}}{2 k^{2}}\left(\left(\frac{r}{i}+\frac{\rho}{i} N k_{k}\right) \varepsilon_{b}+\frac{r}{r} \varepsilon_{i}\right]
\end{aligned}
$$

where $\mathrm{k}$ is the wave number and $\mathrm{u}=\mathrm{kS} \Gamma^{-1} \mathrm{v}_{\mathrm{Sb}}$ where $\mathrm{v}_{\mathrm{Sb}}$ is the gauge-invariant quantity for the baryonic velocity perturbations.

The analogous equations for perturbations of $R$ matter are much more complicated, however, as we can see these perturbations, $\varepsilon_{R}$, come to the equations (4) only as a simple source term. This suggests a simple approximation. Let's assume that $R$ perturbations switch on in the moment $t=\Gamma^{-{ }^{\prime}}$ with the same amplitude as $X$ perturbations that give them the birth. Then they evolve as the growing mode of perturbations of radiation in radiation-dominated universe and decay instantanuously when they enter the horizon. These means that for $x<1 \varepsilon_{R}=0$, for $x \geqslant 1$ (Bardeen 1980):

$$
\varepsilon_{R}=\varepsilon_{b}(x=1) \cdot \frac{y j_{1}|y|}{\left.y_{1} j_{1} \mid y_{n}\right)}
$$

where $j_{1}(y)$ is the spherical Bessel function of order 1 , $y=k \tau c_{s_{2}}, \quad \tau=\int_{0}^{x}(r s)^{-1} d x, y_{1}=y(x=1)$ and $c_{s}$ is the speed of sound, $c_{s}^{2}=1 / 3$. For a given $k$ again is $\varepsilon_{R}=0$ for $\tau \geqslant 3 \pi / 2 k$ (this value was choosen because then the radiation perturbations stop to grow and start to decay). 
c. The MBR anisotropy.

The anisotropy of MBR on large scales comes from the perturbations of geometry that can be conveniently expressed as functions of baryonic perturbations and rave to be integrated along null geodesics. We are interested in the value of the quadrupole moment that is given by:

$$
\left(a_{2}\right)^{2}=4 \pi \int_{0}^{k_{\max }} k^{2}\left|A_{2}\right| k||^{2} d k
$$

where:

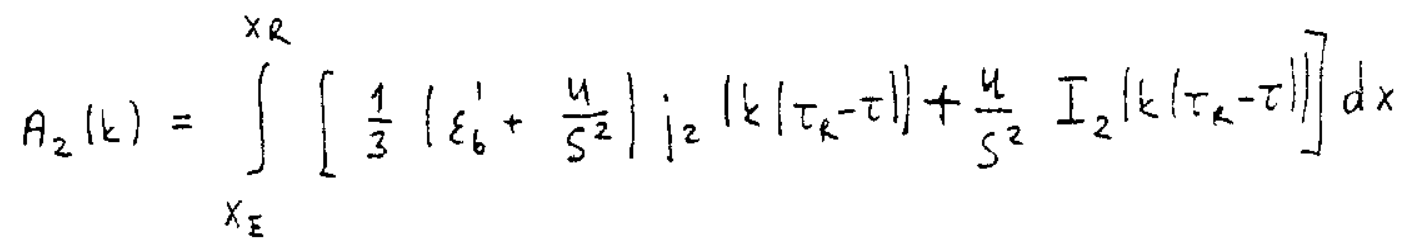

The subscripts $E$ and $R$ denote emission and reception (we assumed that emmision occures at the redshift $z_{E}=13001, k_{\max }$ is the scale corresponding to the size of the horizon at emmision (in fact all the power comes to $a_{2}$ from scales $k<0.2 k_{\max }$ ) and the function $I_{2}$ is equal to:

$$
\left.I_{2}|y|=\frac{1}{5}\left|\frac{2}{3} j_{0}\right| y\left|-\frac{55}{21} j_{2}\right| y\left|+\frac{12}{7} j_{4}\right| y \mid\right]
$$

The procedure was then as follows: first, to solve the equations for the background (3), second, use the solutions to integrate the equations of perturbations (4) and third, use the perturbations to obtain the value of quadrupole moment $(6),(7),(8)$. 
III. THE NORMALIZATION OF SPECTRUM OF DERTURBATIONS.

The inflation gives us not only the flat universe, but also a detailed form of the spectrum of initial, adiabatic perturbations. This is the

Harrison-Zeldovich spectrum: $\left|\varepsilon_{j}(k)\right|^{2} \sim k$. Assuming this initial spectrum and following the procedures described at the end of the previous section we obtain the value of quadrupole moment that is scaled by one, so far unknown parameter - the amplitude of perturbations.

To find this amplitude we have to follow the evolution of perturbations until today and compare resulting distribution of matter with the observed distribution of luminuous matter in the Universe.

For different contens of the universe the initial Harrison-zeldovich spectrum evolves into different forms today. For the $\mathrm{X}$ particles being massive neutrinos the spectrum is (White, Frenk and Davis 1983):

$$
\left|\varepsilon_{b}(k)\right|_{R}^{2}=A_{R} k \exp \left(-4.61\left(k / k_{\nu}\right)^{1.5}\right)
$$

where $k_{v}=0.49 \Omega_{R}\left(1+z_{d}\right) h^{2}$ is the characteristic damping scale.

If the $x$ particles were cold relics we have (Davis et al. $1985)$ :

$$
\left|\varepsilon_{6}(k)\right|_{R}^{2}=A_{R} k\left(1+a k+b k^{1.5}+c k^{2}\right)^{-1}
$$

$$
\begin{aligned}
& \text { where } a=1.7\left(\Omega_{R}\left(1+z_{d}\right) h^{2}\right)^{-1}, \quad b=9\left(\Omega_{R}\left(1+z_{d}\right) h^{2}\right)^{-1.5}, \\
& c=\left(\Omega_{R}\left(1+z_{d}\right) h^{2}\right)^{-2} .
\end{aligned}
$$

The characteristic scales in (9) and (10) come from the size of the horizor at the moment when the universe became matter-dominated. 
Because the details of the galaxy formation and the reliability of the normalization procedures are not well known, usually the normalization is the weakest point of MBR fluctuation calculations. Depending whether the dark matter was hot or cold the galaxy formation proceeds in the different manner. Therefore the appriopriate normalization procedures for both scenarios can be different.

The standard normalization of the spectrum is to require the rms fluctuations of mass in a randomly placed sphere to be equal 1 at the scale of $8 \mathrm{~h}^{-1} \mathrm{Mpc}$ (this value comes from the counts of galaxies on large scales, Davis and Peebles 1983):

$$
\frac{\delta M}{M}|r|=\left(\left.\int_{0}^{\infty} k^{2}\left|\varepsilon_{b}(k)\right|_{R}^{2} w|k r| d k\right|^{1 / 2}=1, r=8 h^{-1} M \rho c(11)\right.
$$

where $W(x)$ is the window function, $W(x)=9 x^{-6}(\sin x-x \cos x)^{2}$.

The other way is to find the value of $J_{3}$ integral and compare it with the observed value on a given scale - we have choosen $R=15 \mathrm{~h}^{-1} \mathrm{MpC}$.

$$
J_{3}(R)=\int_{0}^{R} \xi(r) r^{2} d r=550 \mathrm{n}^{-3} \mathrm{Mpc}, R=15 \mathrm{in}^{-1} \mathrm{Mpc}
$$

where the correlation function is related to the power spectrum by:

$$
\xi|r|=\int_{0}^{\infty} k^{2}\left|\varepsilon_{b}(k)\right|_{R}^{2} \frac{\sin k r}{k r} d k
$$

Instead of that, for the neutrinos we can require the first. 
noninear structures to form at the redshift $z_{n I}=3$ (this is because ve observe quasars at this redshift). We find the amplitude $A_{R}$ from the value of rms dispersion of density perturbations:

$$
\left\langle\left|\varepsilon_{b}(k)\right|_{z_{n}}^{2}\right\rangle^{1 / 2}=\left(\left.\int_{0}^{\infty} k^{2}\left|\varepsilon_{b}\right| k||_{z_{n}}^{2} d k\right|^{1 / 2}=0.6\right.
$$

IV. THE RESULTS.

The results are presented on Figures 1 and 2. Many parameters of the model influence the final values and the situation is more complex than in the standard, one-dominant-component models.

The results obtained with the normalizations related to the counts of galaxies are very close for cold relics and for neutrinos because the parts of the spectra that we are sampling are almost identical for both. When we use "neutrinos" in the following discussion we mean neutrino model normalized for the appearance of the nonlinearity at $z_{n 1}=3$.

First of all the difference in behavior of $\mathrm{a}_{2}$ with $z_{\mathrm{d}}$ for neutrinos and for cold relics is because both spectra are normalized in different manner. Some of the effect comes also from the fact that the lack of growth in DPC cosmology is concentrated in late times. The ratio of the growth factors since emmision till $z_{n l}=3$ and since emmision till today is for smal1-scale perturbations (as used in normalization) equal to $1: 3$ for $z_{\mathrm{d}}=2$ and $1: 2$ for $z_{\mathrm{d}}=10$ but the first growth factor is almost unchanged (the ratio in matter-dominated universe is 1:4). For the cold relics the results are as expected - the larger value of $z_{d}$ means the lower growth factor and then the higher 
initial amplitude required to obtain observed structures. This higher initial amplitude results in higher quadrupole moment.

An interesting feature of the model is that we observe here something more than the Sachs-wolfe effect. This is because only for pure matter-dominated universe the term $\sim\left(\varepsilon_{b}^{\prime}+u s^{-2}\right)$ in eq. (7) vanishes and $u s^{-2} \mathrm{dx} \sim \tau \mathrm{d} \tau$ so we can do the integral in (7) by parts and obtain the anisotropy as a function of perturbations at the emmision. In our model this is not the case because of the change of the equation of state after the emmision - the MBR pattern is also influenced by the history of the universe between emmision and today.

Some intuition about the importance of this effect may be gained from the calculations with $\equiv_{R}=0$ for all the time. The normalization in this case is the same because our model for the $R$ perturbations influences only very large scales. Hence the difference in results that we can observe says us something about the effect of the late evolution of very large scales on the MBR.

The results for $a_{2}$ are for $z_{\bar{d}}=2$ almost the same but for $z_{\mathrm{d}}=10$ they are about 25 per cent smaller when $\varepsilon_{R}=0$. That means that if the decay occurs early enough the radiation perturbations arive the barionic perturbations to grow on scales larger than the horizon. The increased geometry perturbations cause higher fluctuations of the MBR through the non-sachs-wolfe effect.

If we change the value of $\Omega_{N R}$ to 0.3 we obtain the results for neutrinos about 20 per cent for $z_{d}=2$ and 10 per cent for $z_{a}=10$ higher than in 0.1 case. For cold relics the situation is different - the change is about 15 per cent for $z_{d}=2$ and 35 per cent for $z_{d}=10$ but in the opposite direction. 
V. CONCLUSIONS.

The observed value of $a_{2}$ is $<10^{-4}$ (90 per cent confidence), as derived from the observations by Efstathiou and Bond (1986).

From Fig.1. we can see that for cold relics for for neutrinos if the $\mathrm{s} / \mathrm{M}$ or $\mathrm{J}_{3}$ normalization is adopted for both normalizations for $h$ in the range $0.3-0.5$ there are values of

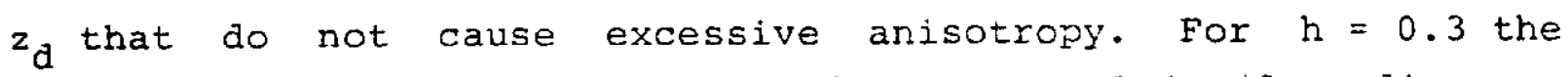
allowed range for the decay redshift is $z_{d}<3-4$ (depending on the choosen normalization). For $h=0.5$ this range is $z_{d}<3-5$. The more detailed treatment for $\varepsilon_{R}$ probably would not produce substantial differences.

For the neutrinos with $z_{n l}=3$ normalization the situation is much worse - any values of $z_{d}$ are excluded. However, without the detailed model of the structure formation we cannot decide which normalization is correct and whether neutrinos can be saved as decaying particle candidates.

The large-scale MBR anisotropy in DPC models is found to be as restrictive as the small-scale one. The range of allowed values of $z_{d}$ found from small scales was < 4 (Vittorio and silk 1985, Kolb, Olive and Vittorio 1986).

If we combine the upper limits obtained above with the lower Iimit $z_{\mathrm{d}}>2$ required to give us sufficiently high value of the ratio $\Omega_{R} / \Omega_{N R}$ today we find the allowed ranges of parameters of the model to be quite narrow. Certainly the normalization techniques have some uncertainity and we should remember of it. Even then the values of $a_{2}$ lie quite close to the observed upper limit. As so far all the measurements of MBR anisotropies maintain the tendency to shift down all the upper limits. The value $a_{2}<10^{-4}$ that we used is quite conservative and we can find observations giving lower values. Recently the value $a_{2}<3 \cdot 10^{-5}$ (95 per cent confidence) was reported (Soviet RELIC 
experiment, as quoted by Kaiser and Silk 1986). If we adopt this measurement we conclude that all the variants of DPC models analysed here give too high values of the MBR quadrupole anisotropy and have to be excluded.

\section{ACKNOWLEDGEMENTS}

Author would like to thank Nicola Vittorio, Roman Juszkiewicz and especially Michael Turner for helpful discussions. This work was supported in part by NASA and DOE at Fermilab. 


\section{REFERENCES}

Bardeen, J.M., 1980, Phys. Rev. D., 22, 1882.

Davis, M., Efstathiou, G., Frenk, C., White, S.D.M., 1985, Ap. J. , 292, 371.

Davis, M., Peebles, P.J.E., 1983, Ap. J., 267, 465. Efstathiou, G., Bond, J.R., 1986, Mon. Not. R. astr. Soc., 218,103 .

Kaiser, N., Silk, J., 1986, Nature, 324, 529.

Kolb, E.W., Olive, K.A., Vittorio, N., 1986, Fermilab preprint $86 / 40-A$.

Panek, M., 1985, Phys. Rev. D, 34, 416.

Turner, M.S., 1985a, Phys. Rev. D, 31, 1212.

Turner, M.S., 1985b, Phys. Rev. Lett., 55, 549.

Turner, M.S., Steigman, G., Krauss, L.M., 1984, Phys. Rev. Lett., $\underline{52}, 2090$.

Vittorio, N., Silk, J., 1985, Phys. Rev. Lett., 54, 2269. White, S.D.M., Frenk, C., Davis, M., 1983, Ap. J. (Lett.), 274, L1. 


\section{FIGURE CAPTIONS}

Figure 1. The values of quadrupole moment $a_{2}$ as a function of the decay redshift $z$ for decaying cold relics(or neutrinos). The triangles are for normalization $6 \mathrm{M} / \mathrm{M}\left(8 \mathrm{t}^{-1} \mathrm{Mpc}\right)=1$ and the circles for $\mathrm{J}_{3}\left(15 \mathrm{~h}^{-1} \mathrm{Mpc}\right)=550 \mathrm{~h}^{-3} \mathrm{Mpc}^{3}$. Open symbols are for $\mathrm{h}=0.5$, filled - for $h=0.3$.

Figure 2. The same as Fig.1. for decaying neutrinos and the normalization $\left\langle\left|\varepsilon_{b}(k)\right|_{z_{n l}=3}^{2}\right)^{1 / 2}=0.6$. Open squares are for $h=0.5$, the filled ones - for $h \stackrel{n}{=} 0.3$. 


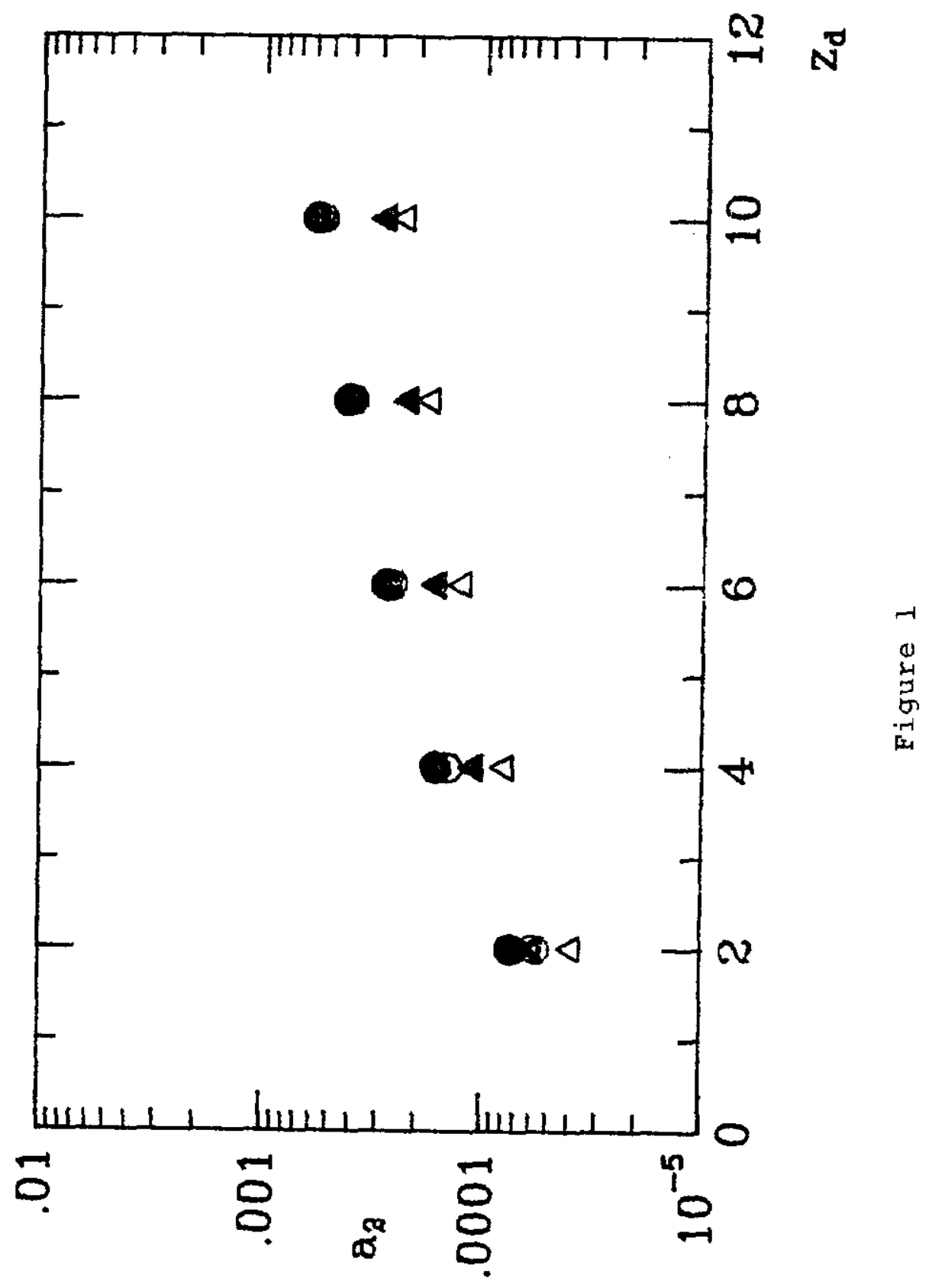




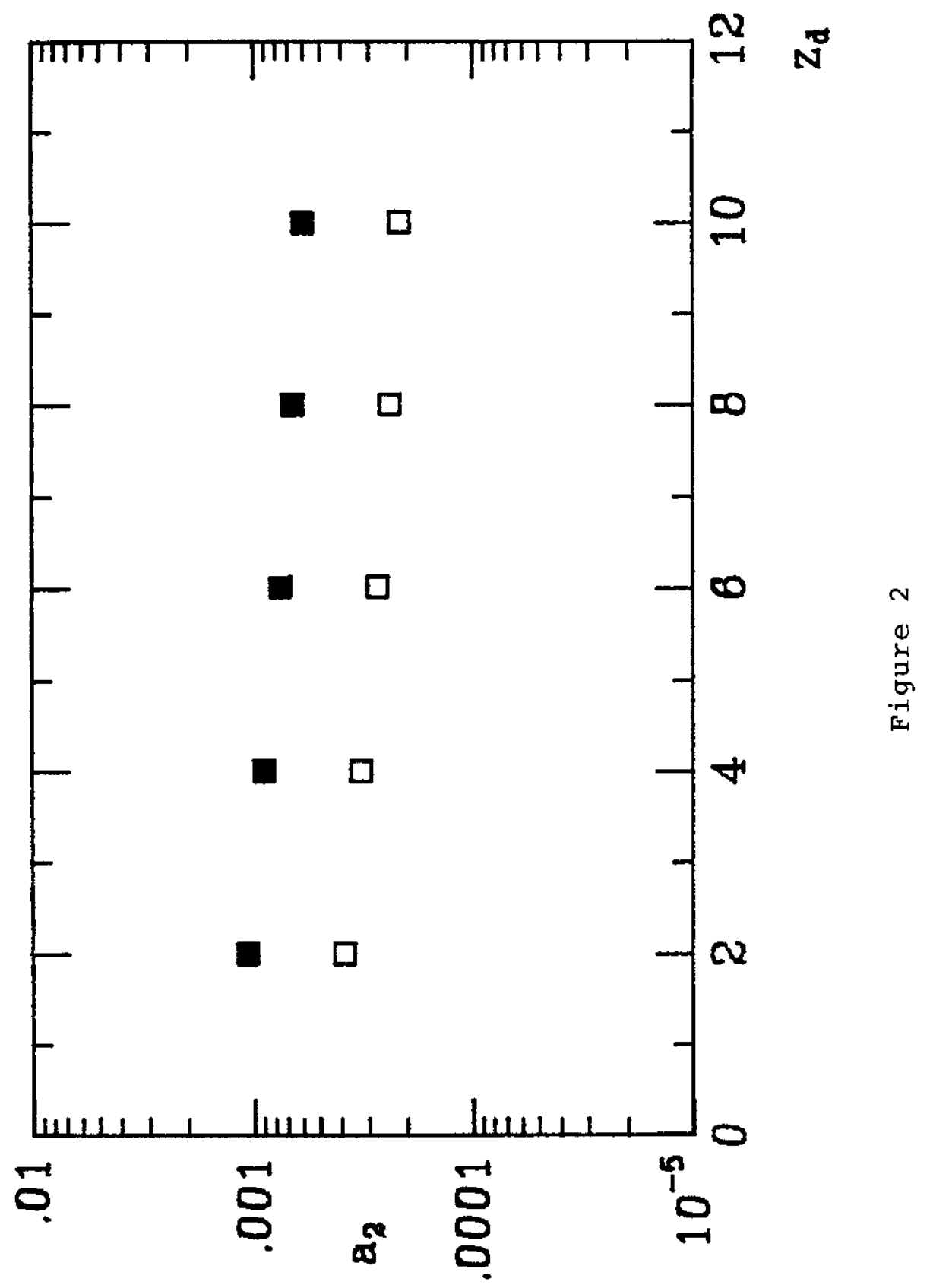

O

$\stackrel{a}{\circ}$ 